\title{
TARGET TRACKING BASED ON BASE STATION NODE USING PREDICTION METHOD AND CLUSTER STRUCTURE IN WIRELESS SENSOR NETWORKS
}

\author{
Farzad Kiani $^{1}$ and Hamidreza Tahmasebirad ${ }^{2}$ \\ ${ }^{I}$ Department of Computer Engineering, Faculty of Engineering and Natural Sciences, Istanbul Sabahttin Zaim University, Turkey \\ ${ }^{2}$ Department of Computer Engineering, Education Technology and Information in Zanjan Province, Iran
}

\begin{abstract}
One of the most important and major challenging issues of wireless sensor networks is the tracking of mobile targets. The network continuously reports the spatial information of moving objects during specified periods to the base station. In this paper, by introducing new a protocol with two versions, of which, one of them is based on dynamic clustering with a focus on the base station, and the other is based on a predictive system for increasing the tracking accuracy of the objects movement and decreasing the energy consumption as well. In this paper, the task of clustering involves in determining the cluster heads, the number of cluster members, the selection of cluster members, and managing the activation of the nodes that is done by the base station. On the other hand, given that the base station is outside the field of wireless sensor networks and is connected to an unlimited power source. The second version of the proposed protocol is based on a predictive algorithm that it was inspired from the first proposed version in the role of the base station node by a prediction method. In this paper, three heuristic models are introduced to select the speed and direction in prediction models. They are instant, average and exponential-average models. These models can track the relevant targets more accurately and reduce the number of missing targets. The simulations are done in different scenarios in a custom developed tool. The results of simulation show a good performance of them in the network lifetime and target tracking applications.
\end{abstract}

Keywords:

Wireless Sensor Networks, Dynamic Clustering, Mobile Target Tracking, Network Lifetime, Target Prediction

\section{INTRODUCTION}

Today, Wireless Sensor Networks (WSNs) are a vital research area and are consist of many small, cheap and limited source sensor nodes that are often working in the distributed environments. In the network environment, sensor nodes sense events via sensing unit, collect and process their data via the processing unit, and transmit them to BS/neighbor nodes via transceiver unit. These networks have self-managing property so they can adapt in different conditions by various protocols. Thus, adaptive protocols implementation is an important issue in network design. For this reason, the resource limitation of sensor nodes is significant factor in the design phase. The designer must have enough information about design factors, communication architecture and stack protocol of the WSNs in designing appropriate protocols. The design factors in the WSN are standard in the protocols implementation. In fact, they play a guideline role and designers can use them even to compare with other models. They are usually reliability, topology, scalability, power consumption, data reports, and transmission media. The WSN stack is consists of five layers and three planes. The layers are physical layer, data link layer, network layer, transport layer, application layer [1]. The planes are power management plane, mobility management plane and task management plane.

The WSNs are widely used in various applications. The first research in this field was inspired by military applications. In addition, they can be used in the fields of urbanization, agriculture, medicine, mines [1-4]. In the network environment, sensor nodes sense events via sensing unit, collect and process their data via the processing unit, and transmit them to $\mathrm{BS} /$ neighbor nodes via transceiver unit. One of the reasons of development and progression of the WSNs is using the inexpensive, small, and affordable sensor nodes [5]. The paper focuses on the use of these networks in detecting and tracking moving objects so it is often used in the military applications [6]. The military applications are many various such as monitoring the war zones, monitoring the forces and operational area of the enemy, collecting information on the arrival of alien forces and the detection of chemical attacks [7]. In addition, the target tracking based applications in the military area can be too much for example, region monitoring and controlling, search for relief, tracking soldiers and cars [7]. The location of the sensor means finding a location with a high precision object is also important as target tracking. Finding the wireless nodes positions and tracking of the mobile targets have become two important areas in the use of wireless sensor networks. Tracking of mobile targets involves finding locations for moving targets using wireless sensors with specific situations.

In the section 2 is discussed the related works about wireless sensor networks and target tracking issue. In the section 3 is introduced a new protocol in two versions that both are based on target tracking applications. The results of simulation and evaluation are explained in the section 4 . Last section presents the conclusion and future works of the paper.

\section{RELATED WORKS}

Most protocols presented in these networks attempt to balance the accuracy between tracking accuracy and energy consumption [8-11]. In these networks, the active keeping of all nodes continuously are cause increasing the tracking accuracy and, of course, maximizing energy consumption in the network. The different protocols presented [12] to solve this problem have tried to avoid waking up sensors that are not involved in the tracking process by using techniques such as selective wake up methods or using predictive approaches to find the next target location. In addition to detecting targets in sensory sensors, another challenge in target tracking is report of the found targets to the Base Station (BS). In protocols that emphasize tracking accuracy and do not adequately track by a single node, clustering or tree formation is the best solution for avoiding energy dissipation and bandwidth. 
In these protocols, an attempt is made to form a tree or a cluster in the region where the target is observed. Then, the data sensors are sent to the cluster head or root of the tree and, after aggregating the related data, transmit to the BS as a single report. It should be noted that these algorithms are repeatedly implemented for the continuous mobility of the target. In fact, the structure of the used tree or cluster in most of the proposed protocol is dynamic. In this paper, the tracking application has been investigated in both aspects. Indeed, target detection with limited number of the sensor nodes and cluster formation to send reports to BS. Therefore, by providing solutions, we will able to solve both issues more effectively than previous protocols. In order to achieve this goal, strategies for designing networks in target tracking should be considered. First of the strategies is instant strategy $[13,14]$. In this model, all sensor nodes are constantly in active mode to monitor their detection area. Under these conditions, objects in the network will always be detected during tracking process and the collected data will be reported to BS with their locations in every $T$ second. Other strategy is monitoring based on synchronizations $[15,16]$. In some applications that do not need to be used to report the momentum of sensed information, this strategy can be used. In this strategy, assuming that all sensor and BS nodes are well synchronized, all sensor nodes can go to asleep and they switch to wake up when it's time to monitor the area of diagnosis and send the report. The other strategy is continuously monitoring [17]. In this model, only active sensors whose targets are in the area where they are diagnosed. Therefore, all sensors in the network do not wake up alternately and do not cover the entire field. An active sensor monitors the target until the target enters the neighboring area. In other words, the node leaves the tracking of the target as soon as that target entries to its neighbors' region. The fourth strategy is dynamic clustering [18]. In this model, the nodes are divided into two categories, the nodes with the highenergy and low-energy. In the forming of each cluster, highenergy nodes are used as Cluster Heads (CHs) and from nodes close to it are used as members of that cluster. The members of the cluster sense the data in the environment and send it to their CHs. The related data will be transmitted to BS after data aggregation by $\mathrm{CHs}$. In this process, the sensor nodes will have spent their batteries, so, the nodes that play more roles in the data sharing process will be discharged the batteries earlier and this is more natural. In this case, management of low-energy nodes and redeployment of the clusters and re-election of $\mathrm{CHs}$ are solutions to be done. To prevent the loss of the target, $\mathrm{CHs}$ should keep the environment steadily. Therefore, the $\mathrm{CH}$ task is more important than the other hierarchy based sensor network applications. The last strategy is based on prediction $[19,20]$. Sensor nodes use this model to predict the next target location. The nodes use the wakeup mechanism to wake up their neighbors before the target exits from the node's sensory area and enters to the node's adjacent region. If the protocol loses the target, the repair algorithm runs over the entire network as alternative solution.

Clustering of sensors is done to facilitate the processing of shared information in tracking targets in the WSNs. The dynamic clustering method is used to cluster formations, $\mathrm{CH}$ elections and management of shared channel. In generally, the characteristics of the cluster include the size of the cluster, the area that it covers it and the number of clusters. In the dynamic clustering methods in the target tracking based applications, many approaches use the classical exist algorithms and methods and the number of new work studies in this area is low. The related protocols must be having several steps to cluster formation and establish links between nodes such as select the $\mathrm{CH}$ nodes, selection of some nodes for target tracking as tracker, selection of nodes to provide information for the newly selected cluster node, and update old tracking information at different times. Each $\mathrm{CH}$ node provides a packet that contains the individual energy and password of the detected signal. In addition, it receives sensor responses for cluster processing, it estimates the target position based on positioning algorithms.

\section{PROPOSED PROTOCOL}

The proposed method is based on a prediction based monitoring model in a hierarchy structure. In this section, we introduce two versions in our protocol for mobile target tracking that in first version, the target tracking and management of it is done by BS node in a hierarchy structure. The second version uses an artificial method based on heuristic models to prediction targets. The model of system is based on a decentralized architecture that the network area is divided to clusters. The network task is target tracking. In each cluster, one sensor node is selected as cluster head. It collects its cluster member data and transmit to base station node in order to data analyzing or monitoring. In this model, role of the base station node is very important so it plays the backbone role in the network. It is introduced in the bellow part of the paper. Then, a method is proposed that it is based on a predictive algorithm. It is inspired from first version and many of its assumptions are based on the TTDCBS. The major difference is that it predicts the movement of the targets based on the heuristic functions. A brief summary of this model is shown in Fig.1.

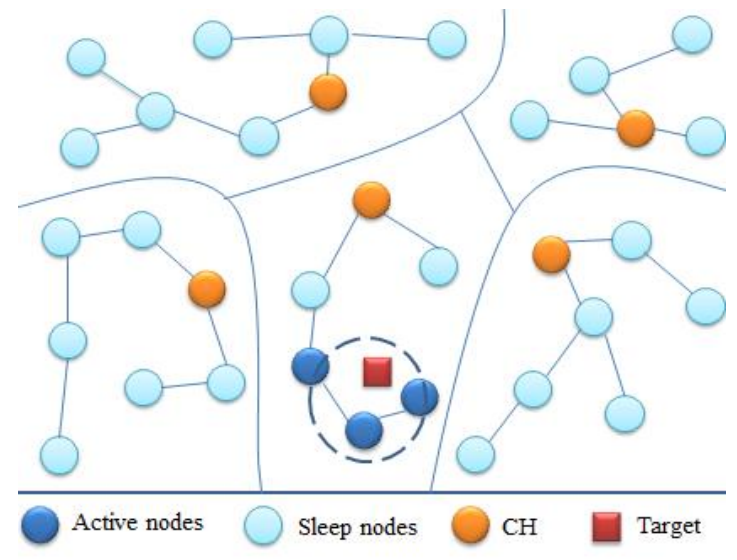

Fig.1. A brief of the proposed model in target tracking based applications

\subsection{FIRST VERSION OF PROPOSED PROTOCOL}

The first proposed version is based on target tracking in dynamic clustering structure with management of base station node (TTDCBS). The main idea is to exchange more calculations against the production of predictive models to save on the number of transmission and in result increasing network lifetime. The energy used to send a packet is, on average, several times the energy consumed in receiving it. On the other hand, consumption energy in receiving a packet is far higher than the amount of 
energy consumed in execute a command. Therefore, reducing the number of high-energy operations is necessary. The proposed method, in addition, transmit the tracked data in each round to the $\mathrm{BS}$, so that the BS is aware of the target current position. Any nodes and CHs aggregate these data, so, the volume of transmitted data to BS will be not high, and will be prevented of the redundant and supererogatory data. Given that the base station is outside the field of wireless sensor networks and is connected to an unlimited power source, it can increase the power of its transmission when needed and send its information to distant distances. By considering this capability and given that the target spatial information is reported to the base station at any one moment, the base station is interfered in the clustering process and it has important role in the network.

Due to the nature of broadcasting in the wireless communications, all sensors are able to hear the information that are transmitted via BS node. In the proposed method, only the sensors receive information that has already been triggered and their status change to awake. The model of wake up is inspired of $[1,27]$ methods as is shown the flowchart of it in Fig.2. Therefore, in the BS transmission, only sensors that are in an awake state consume energy. Since the sensor nodes report the estimated position of the target in specific rounds, the BS can handle a portion of the load of the tracking process with the presence of a wireless sensor network arrangement and the estimated position of the target. In the proposed method, besides this task, it can be used in other operations such as cluster creation and the role tracking process.

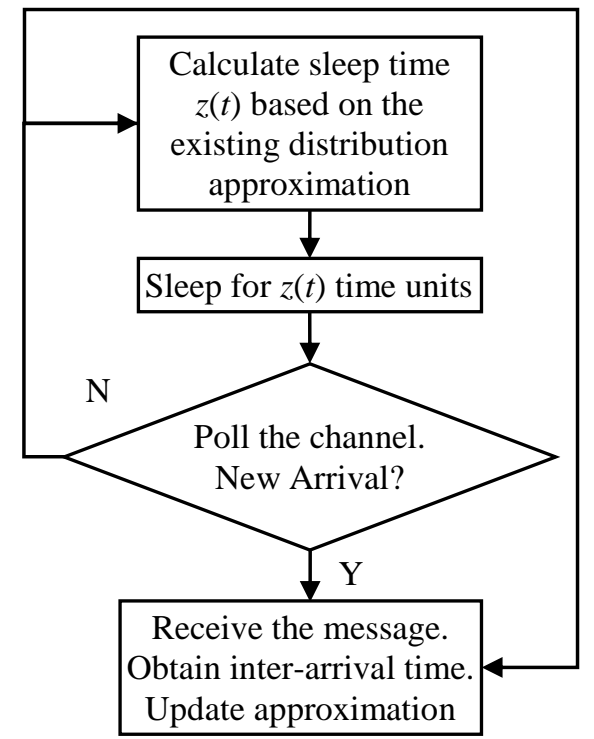

Fig.2. Flow chart of the adaptive dynamic sleep time control [27]

Clustering is one of the required operations in aggregation and fusion. The problem in dynamic clustering algorithms is that clustering must be restarted when the target repeatedly changes its location and it is mobile. Therefore, the energy consumption will be increased and consequently the efficiency of these algorithms will be decreased. In the proposed algorithm of this paper, cluster overhead is reduced by using the base station. In this method, whenever there is a need for re-clustering, the BS determines the new cluster head and new cluster members by sending a "cluster formation" message. The BS selects a node as $\mathrm{CH}$ that it is the nearest node to estimated target position and a number of its neighbors that has in one hop with new $\mathrm{CH}$ node chooses as members of its cluster as is shown in Fig.3.

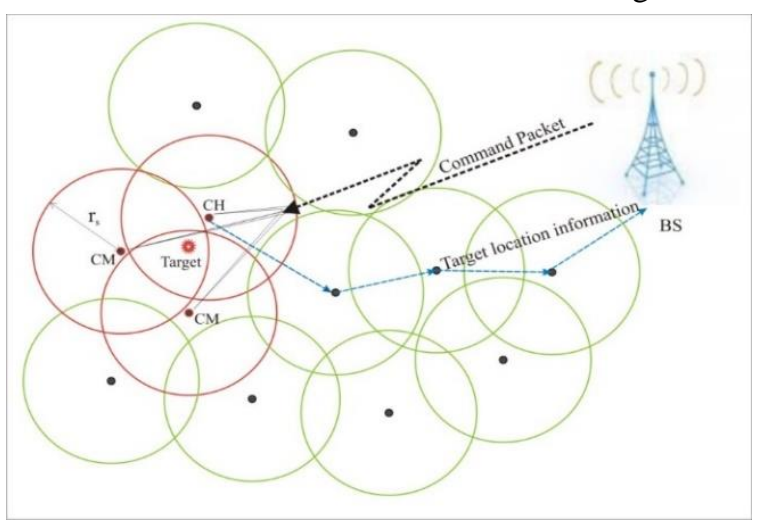

Fig.3. A snapshot of the target tracking [23]

Then, the BS creates a "cluster formation" message containing new cluster information such as cluster identifier and new members and broadcasts it on whole of the wireless sensor network. The base station sends its data in a single hop with high power at a frequency of $868 \mathrm{MHz}$. The sensors receive information from base station by three-dimensional cube antennas as is shown a sample view of it in Fig.4. With this antenna, it is possible to use mixed from facilities of RFID technology and other small and range based wireless devices. The cubic antenna is three-dimensional and very compact with dimensions of $3 \mathrm{~cm} \times 3 \mathrm{~cm} \times 3 \mathrm{~cm}$, the length of each dimension is as large as $\lambda / 11$. The cube shape of the antenna allows the sensor equipment to be cleverly and easily packaged in an empty space inside the cube. As mentioned, their frequency bands are $868 \mathrm{MHz}$ so, both the use of this frequency and the use of a three-dimensional antenna will enable the base station to perform long-term broadcasts quickly and shortly. Therefore, the role of the BS can be bold inside of the network as a backbone. It can be use in the different application areas especially in the target tracking based applications.
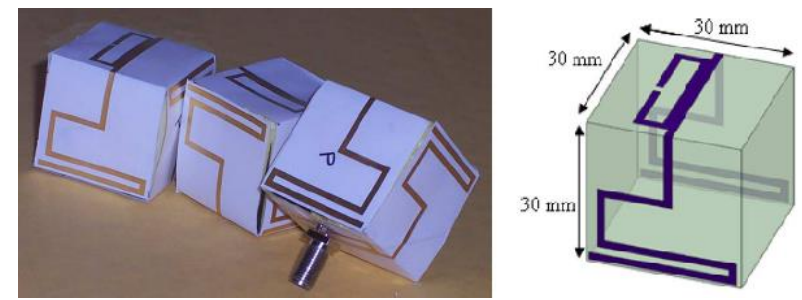

Fig.4. A view of the antenna used in the proposed protocol [18]

Sensors activated in the field receive a clustering message and extract the information and then the clustering phase is completed. The BS determines which sensor should be the new cluster head using the received information from the new target location. Indeed, the proposed method can be used in areas where targets are intense and mobile. The BS announces the $\mathrm{CH}$ and new members to awake sensors. If any error in the sensor node cause to data lost, the recovery operation is called. This operation continues until the BS finds the related target. Therefore, the suggested algorithm has suitable performance in fault tolerance. Since the base station is responsible for clustering and tracking guidance, it has good knowledge about the energy level of each node. It uses this information in the clustering and prevents in 
selection low battery sensor nodes as $\mathrm{CH}$. In addition, it can do not awake some nodes that their energy is in critical level.

The BS calculates the target location after receiving information from the sensor fields. The closest node to the estimated target location is the $\mathrm{CH}$ candidate. However, the energy level of this node should be greater than the threshold $(\alpha)$. If the energy level of the node is less than the threshold, then the nearest next node will be to the estimated target location as the candidate node. Of course, this node should also have a threshold limit. This process continues until a candidate node satisfies the energy condition, otherwise, the BS reduces the amount of $(\alpha)$ to half the current value. By narrowing the area, it can quickly select the $\mathrm{CH}$ and increase the likelihood of the target being caught. Then, the BS again examines the candidate nodes one by one until it finds the suitable cluster head and a number of its neighbors that has in one hop with new $\mathrm{CH}$ node chooses as members of its cluster.

The number of cluster members depends on the need for use, which directly affects tracking accuracy and energy consumption. In the proposed protocol, the number of cluster members in the base station is determined and so, the $\mathrm{BS}$ can reduce the number of cluster members at any moment in the tracking process. It should be noted that the change in the number of cluster members directly effects on tracking accuracy, number of transmission between the nodes of each cluster, and most importantly, on energy consumption.

In the case of the members of each cluster, as well as the $\mathrm{CH}$ selection, the energy condition must be respected and the energy level of the cluster members must be greater than the predetermined value $(\beta)$. The BS will look for $\mathrm{n}$ nodes (number of cluster members) with an energy level higher than $(\beta)$. If it does not find such $n$ nodes, then the value of the threshold $(\beta)$ is reduced by half its current value, and again it tries in finding the $\mathrm{n}$ neighboring node. On the other hand, the proposed algorithm prevents the load balancing problem that is discussed in $[21,22]$ The BS must first wake up the cluster node and the cluster members by trigger and prepare them to receive information in order to receive the clustering message. Because all sensors automatically return to a sleep state after certain periods. It transmits the "cluster formation" message, which includes the new cluster head ID and address, on the wireless sensor network in order to it can introduce the new $\mathrm{CH}$ to nodes. In this protocol, subsequently, other awakened nodes that does not selected as cluster heads can be selected as a $\mathrm{CH}$.

In this protocol, sensory radius of sensors is equal to RS and sensor sensing information by $S_{i}$ is named $S_{i}(T)$ that is calculated by Eq.(1) [23]. The sensor nodes can detect it when the sensor senses the target. Also, $d\left(S_{i}, T\right)$ shows the distance between target node $(T)$ and the sensor node $\left(S_{i}\right)$.

$$
S_{i}(T)=\left\{\begin{array}{l}
1 \text { if } d\left(S_{i}, T\right) \leq R_{s}(i) \\
0 \text { otherwise }
\end{array}\right.
$$

Finding the target position is based on broadcasting that is calculated by Eq.(2). With the assumption that the $T$ target is in the coordinates of $\left[X_{t}, Y_{t}\right]$, all sensors that are located in circuits in the center $\left[X_{t}, Y_{t}\right]$ and Radius $\left(R_{S}\right)$ can detect the target. Generally, the center of gravity of the sensors that can detect the target can be an appropriate estimate for the target location, where, $\left(X_{i}, Y_{i}\right)$ and $n_{s d}$ are the coordinates and number of sensors that identify the mobile target.

$$
\left\{\begin{array}{l}
\bar{X}=\frac{1}{n_{s d}} \sum_{i=1}^{n_{s d}} X_{i} \\
\bar{Y}=\frac{1}{n_{s d}} \sum_{i=1}^{n_{s d}} Y_{i}
\end{array}\right.
$$

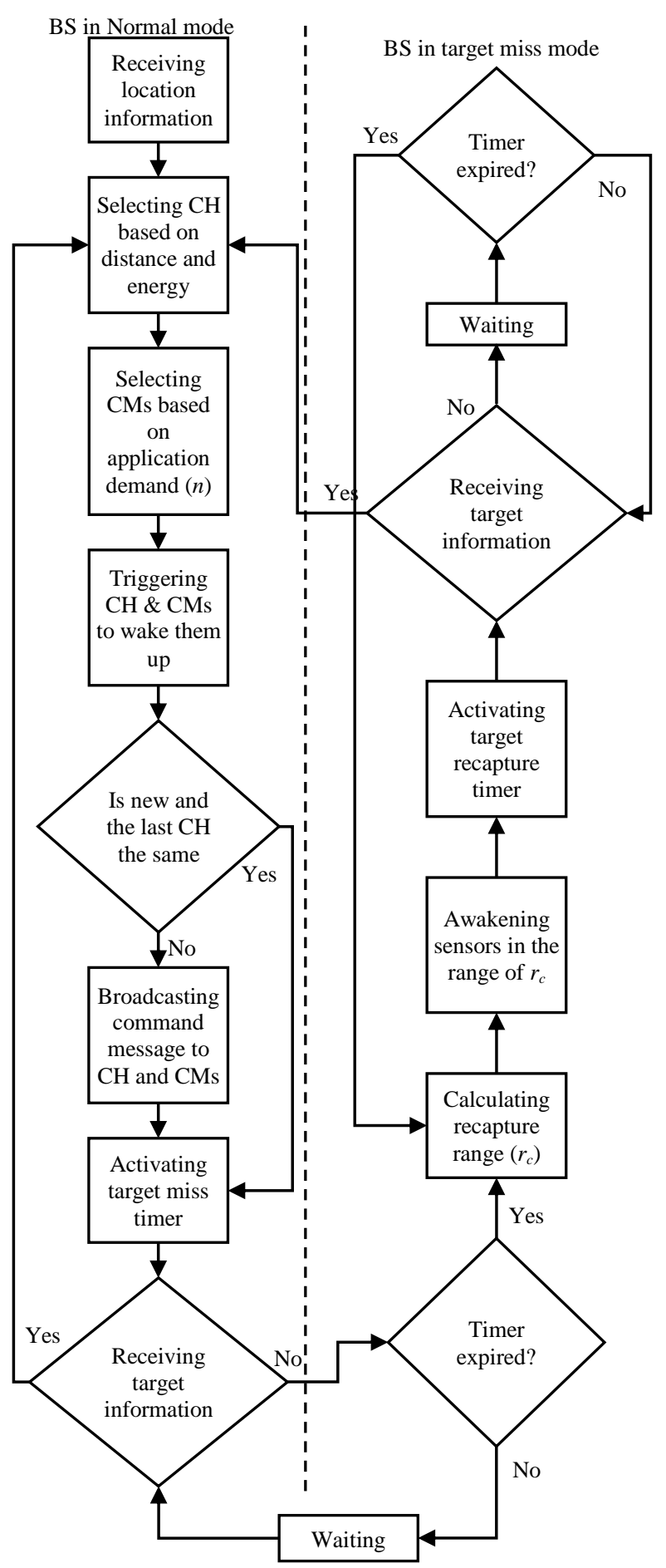

Fig.5. Workflow of proposed method in BS side 


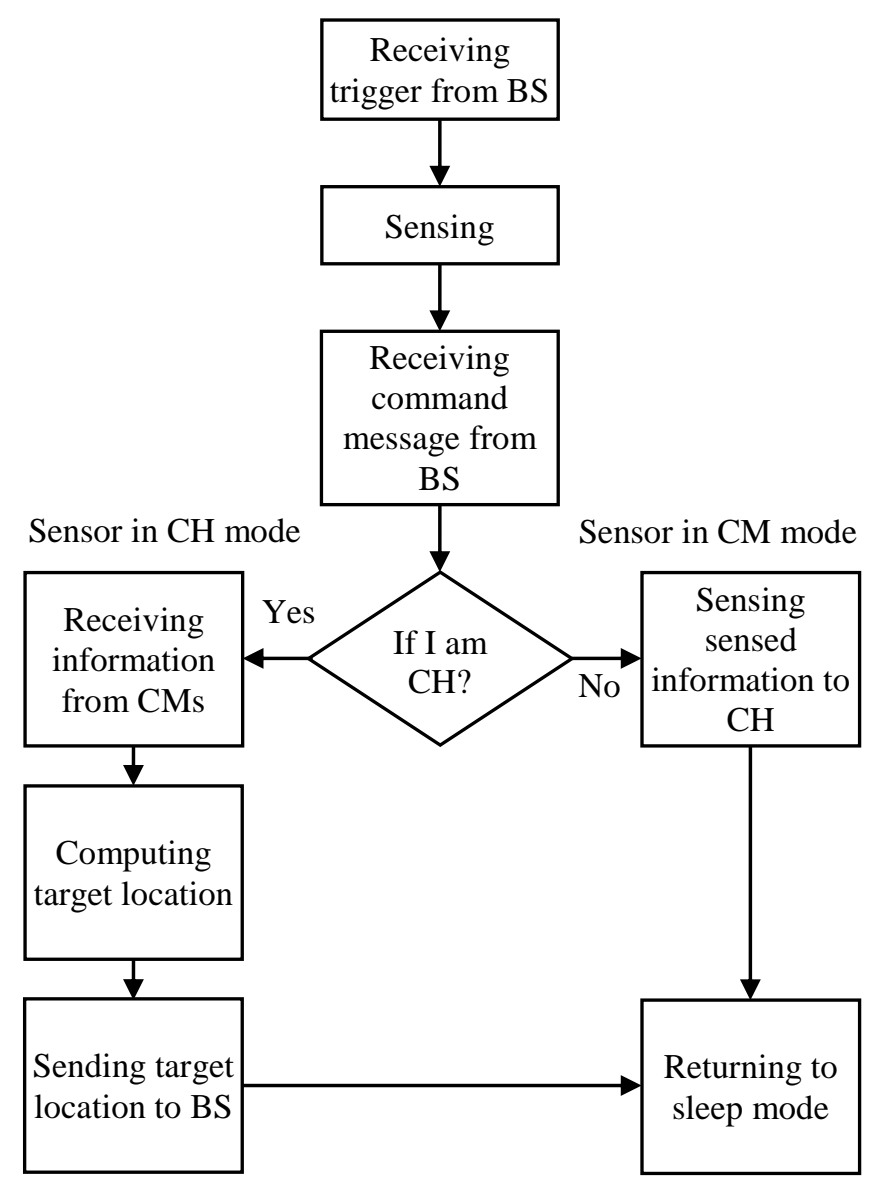

Fig.6. Workflow of proposed method in sensor node side [23]

The Fig.5 and Fig.6 present the steps of the proposed method that the framework in both BS and sensor node side. At the beginning of this algorithm, all nodes are in active state and it is assumed that the target is within the sensory region. When one or more nodes observe the target nodes in their sensory area, they wait for a random time as called back off time in order to avoid a collision in channel and then send the sensed data to the BS node.

\subsection{SECOND VERSION OF PROPOSED PROTOCOL}

On the other hand, in this paper is introduced a predictive algorithm as second version of the proposed protocol. It is inspired from first version and many of its assumptions are based on the TTDCBS. The major difference is that it predicts the movement of the targets based on the heuristic functions. The name of the second version of the proposed protocol is target tracking based on base station node using prediction method (TTBSP).

The prediction results on the movement and destination of the mobile target directly affect the probability of choosing cluster members. If the prediction is correct, a smaller number of cluster members will need to be active, and naturally, the overhead will be reduced. In this section of the paper, an instant prediction model is proposed that the target movement remains constant for a given period (round). Thus, the BS can predict the target movements and destination node. The node that ultimately ends up is called the destination node. These predictions take place regardless of the speed and direction of target movement in this period. Therefore, these factors used in the calculations will differentiate the results of the predictions. In this paper, three heuristic models are introduced to select the speed and direction in prediction models. One of the heuristic methods is instant heuristic. In this method, the BS assumes that the moving target maintains its current speed and current path until the next period. This heuristic is very simple, because the base station does not need to know the history of the target movement. Second method is average-heuristic model. It allows to BS that get the speed and direction of the target for the next period by recording a section of the history of the target movement. In this model, averages of the history are used in calculations. Mostly, the average heuristic method works better than instant heuristic method. The number of records of motion history depends on factors such as the amount of delay and tracking accuracy required for application. Last model is exp-avg heuristic. This heuristic attributes different weights to different stages of motion history. In contrast to previous heuristics, this is not only able to control the weights of a history of motion, but can predict the future outcome of the goal better than the previous two models.

Naturally, in each case, predictive methods are not error-free. In order to correct predictive errors, a set of destination nodes will awaken to help with the target tracking process. A wake-up mechanism that determines the membership of cluster members based on different levels of conservatism is presented in Fig.7.

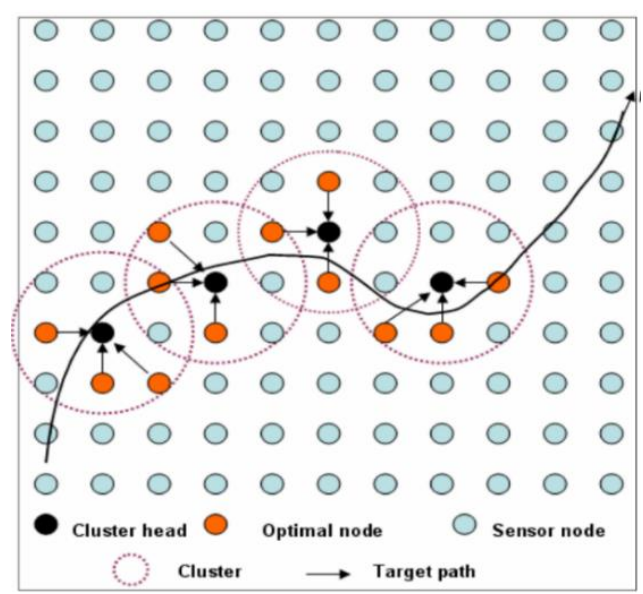

Fig.7. Proposed heuristic models for the wake up mechanism

In the wake up mechanism are also used the three heuristic models. The proposed instant heuristic model is used in destination and BS nodes relations. So, the BS only informs the destination node about target. Therefore, the overhead of this model is just one node, but the probability of missing a target is high. The second model is used as network path heuristic. It includes nodes that are in path of the current node to the destination node expected prediction of destinations. This model assumes that the right direction is guessed, but there is the possibility of error in speed estimation. Therefore, waking up nodes in the path can effectively obtain speed variations. The third model is used in calculation of all neighbors' heuristics. In this case, the BS awakens the nodes that surround the current node to the destination other than the nodes in the destination path. This heuristic assumes that the prediction of velocity and direction values with the real values has an intangible difference. Therefore, waking up the nodes further reduces the chance of losing the target. 
Between these heuristic models, the model based on all neighboring nodes is more conservative than the other models. Because it assumes both the direction and the predicted speed can be error-prone. The destination and path heuristic models are more energy-efficient, because they assume that at least one of the predictions is correct and, as a result, they will wake up with less nodes. It is intuitively clear that, with the increase in the number of active nodes, traceability is performed at a higher quality, and the probability that the moving object will be lost is also reduced. However, increasing the number of nodes in the network means increasing energy consumption. However, remember that if a track of target is loss, it will need to wake up all nodes in a very high-consuming process.

The proposed predictive algorithm is used to identify the CHs that will be located on the target's path according to the heuristic values. Therefore, these cluster heads can use this information to lead of the suitable sensors for target tracking purposes. After the BS predicts the next location of the target, the first node, which is close to the target-oriented site and has the required energy, is awakened as the head of the cluster by proposed predictive algorithm. In addition, it awakes the nodes that are near to selected $\mathrm{CH}$ nodes as new member of the cluster. The number of members of the new cluster is managed by the BS, which has a direct impact on the accuracy of tracking of the target. The new $\mathrm{CH}$ provides information about target location predicted from the BS node and immediately selects three sensors (if possible) such that their distances to the predicted location are less than the normal radius r. After selecting the three sensors, the BS sends them a wake-up message to track the target. If it is not able to find the target in this sensory radius, it must awake other suitable sensors within the radius $\mathrm{R}$. In addition, if it still fails to reach the target in this radius, the $\mathrm{BS}$ awakes the adjacent $\mathrm{CHs}$.

This protocol cannot guarantee that the reports success rate will be $100 \%$, unless it awake all nodes like the scheduled monitoring pattern. In this pattern, you do not need to report sensed information at any moment. Here, it is assumed that all sensor nodes and the base station are well synchronized and all sensors can switch to sleep mode. They will wake up when the time for monitoring the area of diagnosis and reporting has come. Assuming we define every round of network in $T$ seconds, all sensor nodes are activated for $X$ seconds and then will be switched to sleep mode for $(T-X)$ seconds.

When ACK packets or target is lost, data retrieval can be important in the shortest time with minimal energy consumption. The proposed retrieval algorithm calls the tracking operations that has faced with failure. The BS attempts to calculate the target recovery board $\left(R_{c}\right)$ and wakes up all the nodes in this interval. When the BS completes the "cluster formation" message, the counter of the target's loss will activate, and thus the target retrieval process begins. If a base station does not receive any report from the $\mathrm{CH}$ before the expiry of the counter, a target lost event has occurred. In this case, $R_{c}$ is calculated based on the latest speed and maximum target acceleration. The BS awakens all the nodes in the radius $\left(R_{c}\right)$ coverage, but no message is sent to the sensors field. The meaning of this status is considered to be the "loss of target". If the BS succeeds in recovering, the normal tracking process is restored, otherwise the recovery radius doubles to its current value and the BS awakens the nodes within the new radius and will seek the target. This process continues until the main station reaches the target or the network life is over.

The network sensors, as soon as they receive the wake-up trigger from the BS, change their state from sleep to awake and wait for the message "cluster formation". If the sensors do not receive any messages, each sensor detects the target in its sensory range and separately sends related data to the BS in a multi-hop transmission method. Due to the ease of clustering in the proposed method, the algorithm presented in wireless sensor environments is much more efficient and energy efficient. In addition, it has a good performance in the target tracking so the probability of missing the target is very low.

\section{SIMULATION AND EVALUATION}

Simulation and evaluation of the results for the both proposed methods have been evaluated in two groups. In the first part, the effect of increasing the number of nodes on different simulation parameters has been investigated. The proposed methods (TTBSP and TTDCBS) are compared with the DCTC [8] protocol. The reason why DCTC is chosen for comparison is that, firstly, this protocol has a very good performance in target tracking in hierarchical based networks. Another reason is the similarity of the algorithm to our work in terms of clustering structure. In the second part, the number of cluster members and its effects on the simulation parameters have been considered. In this section, the number of nodes is constant. In the simulations, distribution of nodes is random. In addition, each simulation scenario is repeated 1000 times and in each repetition of the experiment, the sensor nodes are in a different layout than the previous layout.

We also developed the required simulation software in the $\mathrm{C \#}$ environment to evaluate the validity of the proposed algorithms and to compare these protocols. The Fig. 8 shows a snapshot of the implemented program as simulation tool. In the implementation, the possibility of a collision between the packets is not considered because the tracking application at any moment only involves a small area of the sensor network field in the process of processing. In simulations, the movement model is a Random Way Point (RWP) [24]. In general, this model is used in the target tracking based applications. In implementing this model, the movement speed of the target can be random and constant. However, after stopping at a point and moving to the next random point, the target speed can be varied by $4.6 \pm \mathrm{m} / \mathrm{s} 2$. Maximum target speed is $20 \mathrm{~m} / \mathrm{s}$.

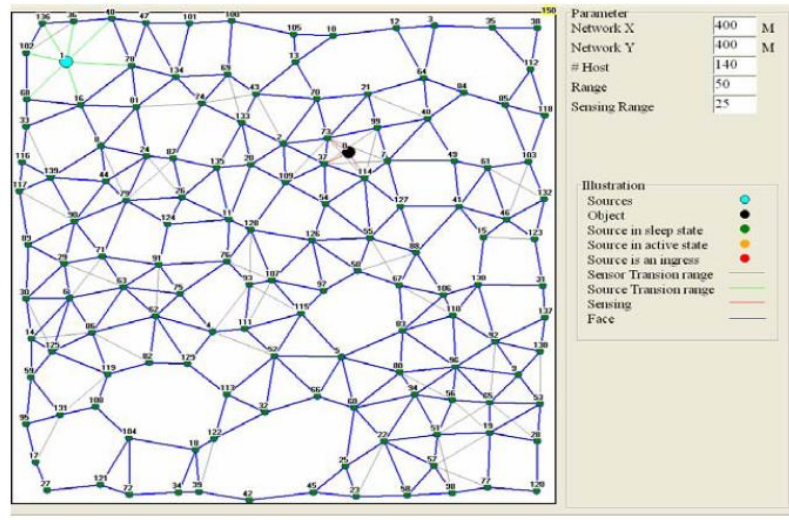

Fig.8. Custom Simulation Platform 
In this study, we simulated a wireless sensor network, including a set of sensors that were randomly developed in an area of $400 \times 400$ square meters. It is assumed that the BS is located at the point $=(0,0)(X, Y)$ and away from the network of sensors. In addition, considering that the location of the algorithms can be calculated by calculating the nodes, it is assumed that the BS is aware of the location of the sensor nodes. Each sensor node is able to detect a moving target within its sensory range. It can also communicate with neighboring nodes within the communication board, and can perform simple calculations. In this paper, the sensory band Rs is $25 \mathrm{~m}$ and the $R_{c}$ is $50 \mathrm{~m}$. The target is moving at a maximum velocity $V_{\max }=20 \mathrm{~m} / \mathrm{s}$ in accordance with the RWP. The initial energy of each sensor is $3 \mathrm{~J}$, the energy consumption in the send state is $0.175 \mathrm{~J}$, and in the receive mode is $0.035 \mathrm{~J}$ and in the sensing mode is $1.75 \mu$ Joule. All these values are taken after studying and comparing several valid papers from [25]. The related parameters and its assumptions are shown in Table.1.

To prove the efficiency, the methods are compared with the DCTC tracking method from the point of view of network lifetime, tracking error, and the number of missing targets.

Table.1. Radio specification and its parameter values for simulating proposed protocols [26].

\begin{tabular}{|c|c|c|}
\hline Vocabulary & Parameter & Value \\
\hline $\begin{array}{c}\text { Threshold } \\
\text { distance for } \\
\text { Friis release } \\
\text { model and two- } \\
\text { ray diffusion } \\
\text { model }\end{array}$ & $d_{\text {cross-over }}$ & $\frac{4 \pi h_{r} h_{t}}{\lambda}$ \\
\hline Transmit Power & $P_{t}$ & $\begin{array}{l}\in \text { friis }- \text { amp } R_{b} d^{2}: d<d_{\text {crossover }} \\
\in \text { two-ray - amp } R_{b} d^{4}: d \geq d_{\text {crossover }}\end{array}$ \\
\hline Receive Power & $P_{r}$ & $\begin{array}{l}\in \text { friis - amp } \frac{R_{b} G_{t} G_{r} \lambda^{2}}{(4 \pi)^{2}}: d<d_{\text {crossover }} \\
\in \text { two-ray-amp } R_{b} G_{t} G_{r} h_{t}^{2} h_{r}^{2}: d \geq d_{\text {crossover }}\end{array}$ \\
\hline $\begin{array}{l}\text { The minimum } \\
\text { amount of } \\
\text { money you can } \\
\text { get to succeed }\end{array}$ & $P_{r \text {-threshold }}$ & $6.3 \mathrm{nW}$ \\
\hline $\begin{array}{c}\text { Power of } \\
\text { amplifier radio }\end{array}$ & $\begin{array}{l}\in \text { friis-amp } \\
\in \text { two-ray- } \\
\quad \text { amp }\end{array}$ & $\begin{array}{c}\frac{P_{r-\text { threshold }}(4 \pi)^{2}}{R_{b} G_{t} G_{r} \lambda^{2}} \\
\frac{P_{r-\text { threshold }}}{R_{b} G_{t} G_{r} h_{t}^{2} h_{r}^{2}}\end{array}$ \\
\hline $\begin{array}{c}\text { Energy of } \\
\text { electrical radio }\end{array}$ & $E_{\text {elec }}$ & $50 \mathrm{~nJ} / \mathrm{bit}$ \\
\hline $\begin{array}{l}\text { Computational } \\
\text { energy for wave } \\
\text { formation }\end{array}$ & $E_{B F}$ & $5 \mathrm{~nJ} / \mathrm{bit}$ \\
\hline Bit rate & $R_{b}$ & $1 \mathrm{Mbps}$ \\
\hline Antenna & $G_{t}, G_{r}$ & 1 \\
\hline Antenna height & $h_{t}, h_{r}$ & $1.5 \mathrm{~m}$ \\
\hline $\begin{array}{c}\text { Signal } \\
\text { Wavelength }\end{array}$ & $\lambda$ & $0.325 \mathrm{~m}$ \\
\hline
\end{tabular}

\subsection{EVALUATION OF NETWORK LIFETIME PARAMETER}

The Fig.9 shows the effect of increasing the number of nodes on the network lifetime. The number of cluster members in this case is assumed three nodes. In both proposed methods, network lifetime increases with increasing number of nodes. However, in the DCTC, the increase in the number of nodes initially increases network lifetime, but after several steps, the network life span will show a downward trend. The downside of the DCTC method is that this protocol firstly pays a very high cost for clustering and most clustering overhead is on the cluster head. With increasing number of nodes, their density and number of neighbors will be increased. When performing clustering operations, $\mathrm{CH}$ has to communicate with its neighbors to select cluster members. Indeed, increasing the number of nodes increases the number of neighbors of a node and, as a result, increases cluster overhead. This increase in overhead, which is largely borne by the cluster head, can be cause to death of the cluster, decrease the network life, and even network disconnection. However, in the proposed methods, increasing the number of nodes increases network lifetime. Since, these methods are more intelligent in choosing the $\mathrm{CHs}$ because of the relative awareness of the BS of the amount of energy consumed and the remainder in the network nodes. In these methods, the amount of residual energy in the nodes is involved in the selection of the cluster. In fact, these two methods use a definite method to determine the $\mathrm{CHs}$ selection and the cost of it related with the BS. The TTBSP protocol has a longer lifetime than TTDCBS, due to it has a good performance in the prediction of the target location, reducing the number of missing targets, and reducing the number of clustering algorithms. In fact, the TTBSP is improved version of the TTDCBS that uses the intelligent metrics in order to predictions.

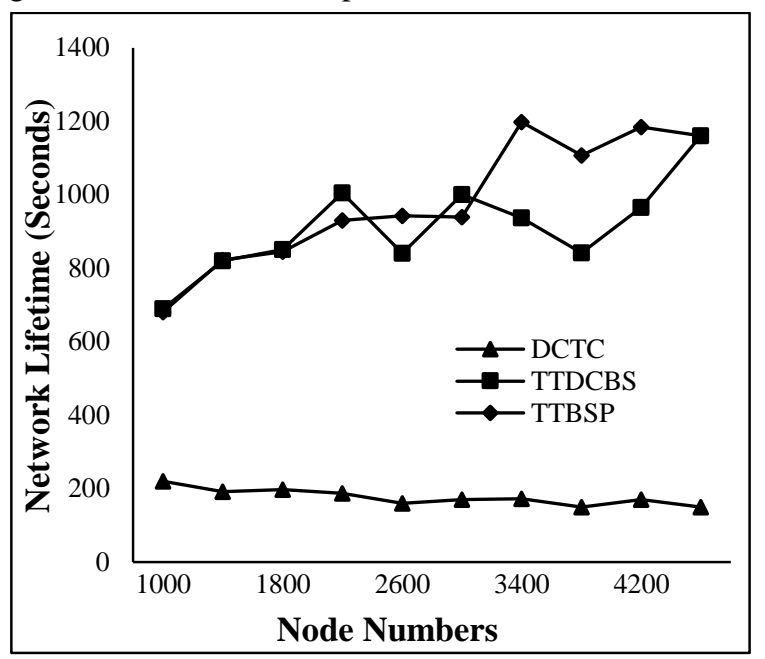

Fig.9. The effect of increasing the number of nodes on the network lifetime as seconds (cluster members $=3$ )

The Fig.10 shows the network lifetime result while the valid cluster members are five. The results show that increasing the number of cluster members has a negative effect on the lifetime of the network. Increasing the number of cluster members from both sides neutralizes the effect of increasing the number of nodes. Firstly, increasing the number of cluster members increases the clustered overhead. Secondly, increasing the 
number of cluster members increases cluster loading while receiving spatial information from cluster members. It is natural that as the number of cluster members increases, the number of packets sent to the cluster head containing spatial information increases.

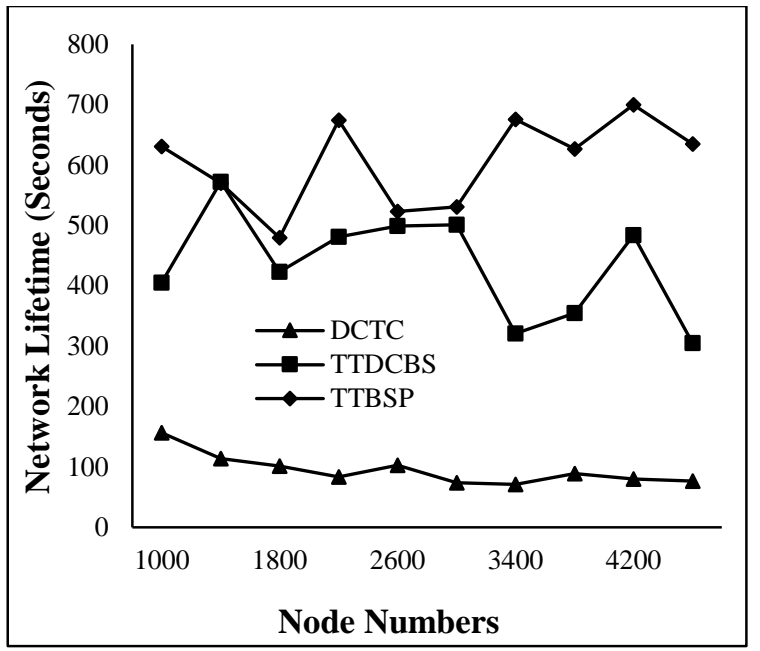

Fig.10. The effect of increasing the number of nodes on the network lifetime as seconds (cluster members $=5$ )

\subsection{EVALUATION OF TRACKING ERROR RATE}

The tracking error is one of the parameters that is specific to the use of target tacking problems as military applications. In order to achieve high tracking and low error rates, the number of nodes involved in the tracking process should be increased. However, means of the increasing the number of nodes involved in the tracking phase is increasing the number of cluster members. As a result, it leads to increase of energy consumption and subsequently reduce network lifetime. Therefore, a balance should be made between minimizing tracking error rate and increasing network lifetime. Two of the proposed methods have been able to illustrate the near-tracking error rate to the existing method (DCTC) rate with very little energy consumption especially the TTBSP protocol. Of course, the error difference is very low and can be ignored. The tracking error results of the three methods are presented in Fig.11 and Fig.12.

Fig.11. The effect of increasing the number of nodes on the tracking error (cluster members $=3$ )

As the number of cluster members increases, the accuracy of predictions increases, and as a result, the tracking error rates are decreased in the proposed methods. In addition, increasing the number of nodes in the network increases network density so it causes increasing the precision of tracking, In other words, reducing the tracking error rate.

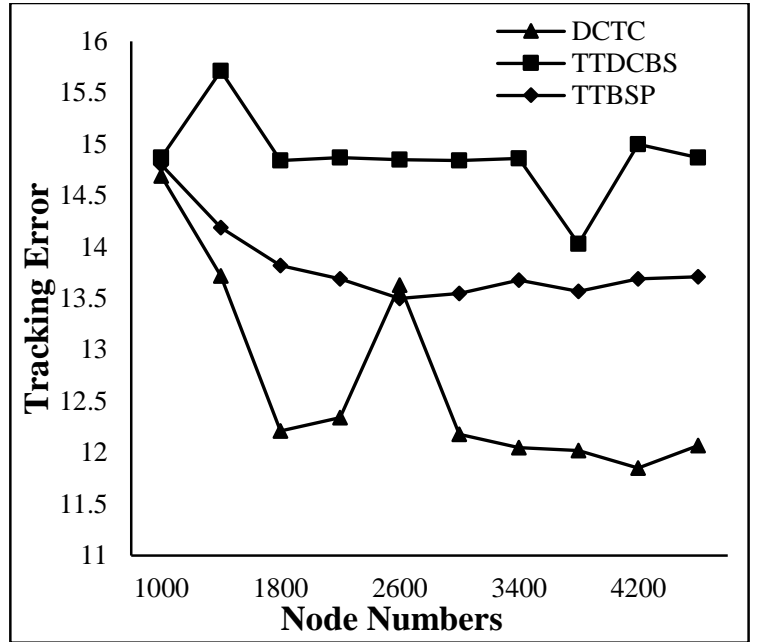

Fig.12. The effect of increasing the number of nodes on the tracking error (cluster members $=5$ )

\subsection{EVALUATION OF NUMBER OF MISSING TARGETS}

The parameter of the number of missing targets is the same as the intercept error parameter of the parameters for the tracking application and is important in the various applications of the target tracking. In rescue applications and in general for applications that are vulnerable to missing targets, this parameter should be kept as low as possible. Given the fact that the number of missing targets is a parameter that should be evaluated in a unit time, the simulation results are presented in two modes.

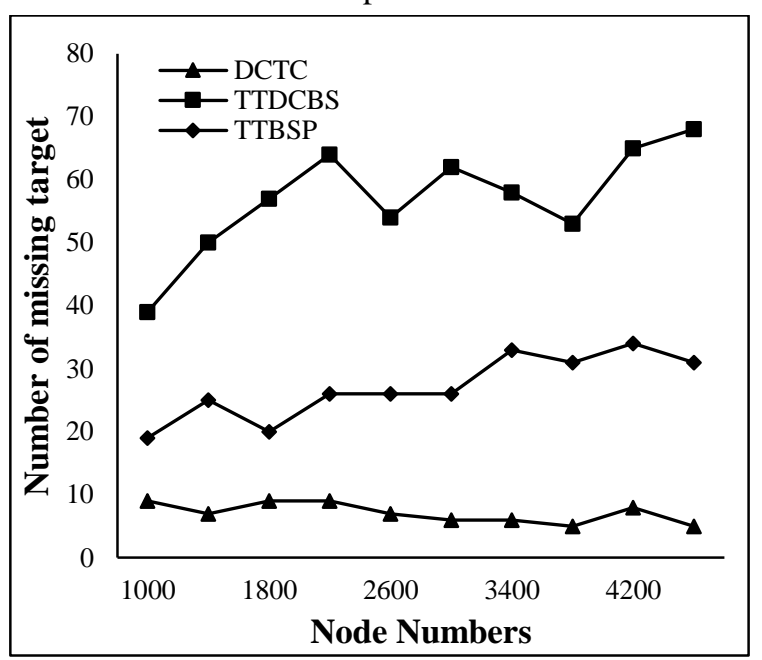

Fig.13. The effect of increasing the number of nodes on the number of missing targets (cluster members $=3$ )

In the Fig.13, the effect of the increasing node numbers is shown on the number missing target parameter. This figure does not compare the methods with each other and only provides information about the number of missing targets in each of the methods. It provides good information for comparing methods. The comparison of the methods performance is presented in Fig.14. The result is shown that the TTBSP protocol has a better performance than the DCTC protocol and the TTDCBS protocol, from the perspective of the number of targets lost in time units. Because it has the ability to predict. Increasing the number of 
nodes did not have much effect on reducing or increasing the number of missing targets. Because, it does not affect the number of cluster members.

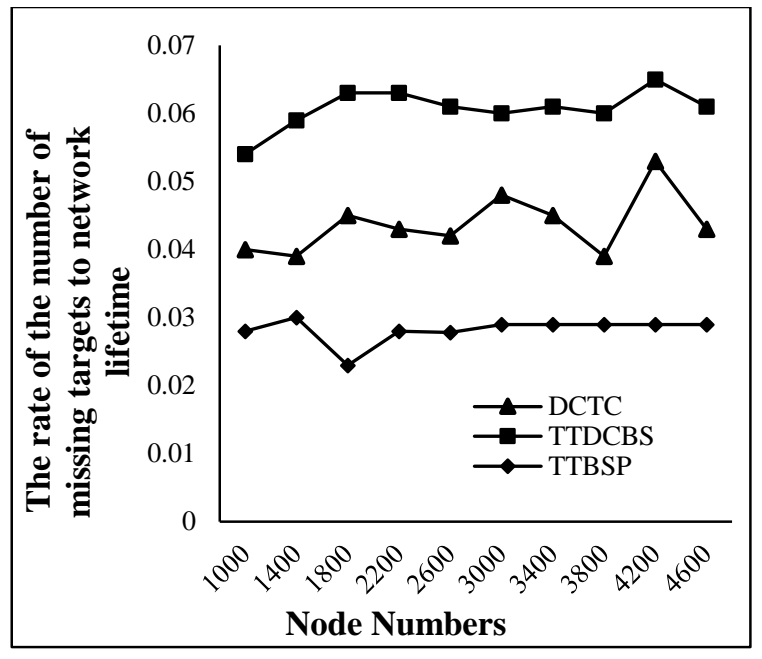

Fig.14. The effect of increasing the number of nodes on the number of missing targets in unit time (cluster members $=3$ )

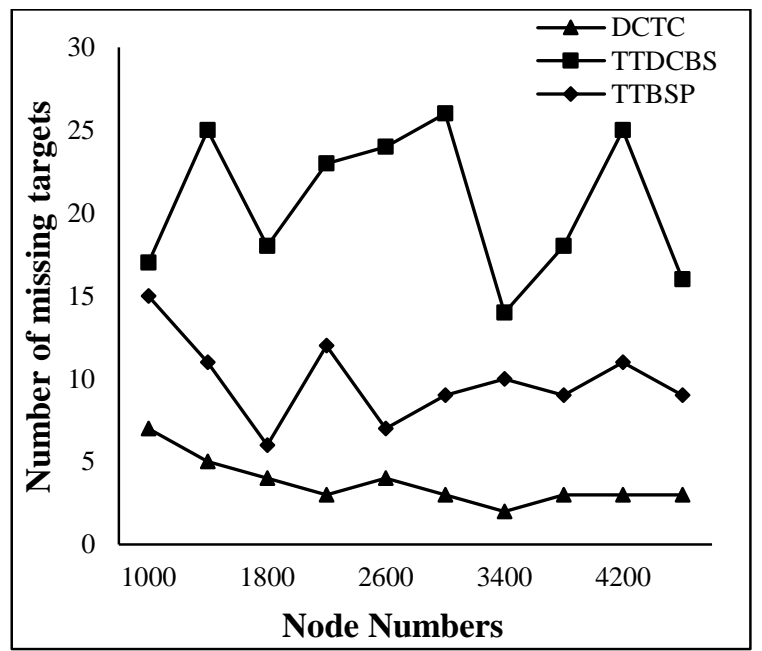

Fig.15. The effect of increasing the number of nodes on the number of missing targets (cluster members $=5$ )

Table.2. Comparison of the efficiency of different methods in improving evaluation criteria

\begin{tabular}{|c|c|c|c|}
\hline Evaluation parameter & DCTC & TTDCBS & TTBSP \\
\hline Network Lifetime & Low & Medium & High \\
\hline Tracking error rate & High & Low & Medium \\
\hline Number of missing targets & Medium & High & Low \\
\hline
\end{tabular}

By increasing the number of cluster members from 3 nodes to 5 nodes, it can be seen that the number of missing targets decreases rapidly. The simulation results are shown in Fig.15. Since the number of nodes involved in tracking depends on the number of nodes in the cluster members, increasing the number of nodes still does not have much effect on the number of missing targets. By increasing the number of cluster members, a significant reduction in the number of missing targets was observed.
By increasing the number of cluster members, the number of missing targets decreases sharply, and also, the results of the two DCTC and TTDCBS methods start to look alike. In this section, the abstract of the simulation results is presented in Table.2.

\section{CONCLUSION AND FUTURE WORKS}

In this paper two methods were proposed in the mobile target tracking based applications. They were simulated in custom simulation tool and were compared with the DCTC protocol in three different parameters as network lifetime, tracking error rate and number of missing targets. The first version of the proposed protocol was on dynamic clustering structure based on management of base station node (TTDCBS). Its main idea was to exchange more calculations against the production of predictive models to save on the number of transmission and eventually increasing network lifetime. The second protocol was based on a predictive algorithm that it was inspired from first proposed protocol in the role of the base station node. The name of the second version of the proposed protocol was target tracking based on base station node using prediction method (TTBSP). It was inspired from the first version and many of its assumptions were based on the TTDCBS. The major difference was that it predicted the movement of the targets based on the heuristic functions.

The prediction results on the movement and destination of the mobile target directly affect the probability of choosing cluster members. Both proposed methods use the base station in the tracking process to reduce the number of transmission messages for cluster formation. In addition, they use of new antennas in sensor networks to provide the ability to receive information from distant distances. In addition, in the second protocol, more complex predictive algorithms and recovery algorithms were used in case of target loss. The simulation results show that the TTDCBS and TTBSP methods have improved the most important parameters of the intercept sensor networks. Of course, with regard to the interpolation accuracy parameter, the existing algorithm (DCTC) is slightly better than the TTDCBS, which is a discrepancy that can be ignored.

There are still problems with precise prediction of the target movement which should be studied in the future. The present study focuses on the tracking of a single target at any moment. In the future, taking into account the target classification techniques; one can extend the work to track several targets in the wireless sensor networks. In addition, it can increase the accuracy of predictive algorithms using more intelligent predictive algorithms and especially artificial intelligence algorithms.

\section{REFERENCES}

[1] F. Kiani, A. Rad, M.K. Sis, A. Kut and A. Alpkocak, "EEAR: An Energy Effective-Accuracy Routing Algorithm for Wireless Sensor Networks", Life Science Journal, Vol. 10, No. 2, pp. 39-45, 2013.

[2] X.Q. Yu, Z.L. Zhang and W.T. Han, "Evaluation of Communication in Wireless Underground Sensor Networks", IOP Conference Series: Earth and Environmental Science, Vol. 69, pp. 1-8, 2017. 
[3] M. Adam, M. Anisi, I. Ali, "Object Tracking Sensor Networks in Smart Cities: Taxonomy, Architecture, Applications, Research Challenges and Future Directions", Future Generation Computer Systems, pp. 1-15, 2017.

[4] H. Jawad et al., "Energy-Efficient Wireless Sensor Networks for Precision Agriculture: A Review", Sensors, Vol. 17, No. 8, pp. 1-45, 2017.

[5] F. Kiani, "AR-RBFS: Aware-Routing Protocol Based on Recursive Best-First Search Algorithm for Wireless Sensor Networks", Journal of Sensors, Vol. 2016, pp. 1-10, 2016.

[6] T.A. Alhmiedat and S.H. Yang, "A Survey: Localization and Tracking Mobile Targets through Wireless Sensors Network", Proceedings of $8^{\text {th }}$ Annual Network Symposium, pp. 2-9, 2007.

[7] M. Akter, "Energy-Efficient Tracking and Localization of Objects in Wireless Sensor Networks", IEEE Access, Vol. 6, pp. 17165-17177, 2018.

[8] W. Zhang and G. Cao, "DCTC: Dynamic Convoy TreeBased Collaboration for Target Tracking in Sensor Networks", IEEE Transactions on Wireless Communications, Vol. 3, No. 5, pp. 1689-1701, 2004.

[9] H.T. Kung and D. Vlah, "Efficient Location Tracking using Sensor Networks", Proceedings of IEEE Wireless Communications and Networking Conference, pp. 1-8, 2003.

[10] F. Kiyani, H. Chalangari and S. Yari, "DCSE: A Dynamic Clustering for Saving Energy in Wireless Sensor Network", Proceedings of IEEE International Conference on Communication Software and Networks, pp. 13-17, 2010.

[11] H. Ahmadi, F. Viani and R. Bouallegue, "An Accurate Prediction Method for Moving Target Localization and Tracking in Wireless Sensor Networks", Ad Hoc Networks, Vol. 70, pp. 14-22, 2018.

[12] F. Kiani, "Designing New Routing Algorithms Optimized for Wireless Sensor Network", Academic Publishing, 2014.

[13] A. Ali, Y. Ming, S. Chakraborty and S. Irem, "A Comprehensive Survey on Real-Time Applications of WSN", Future Internet, Vol. 9, No. 77, pp. 1-22, 2017.

[14] R. Shorey, A. Ananda, M. Chan and W. Ooi, "Mobile, Wireless and Sensor Networks", Wiley-Blackwell, 2006.

[15] M. Navia, J. Campelo and A. Bonastre, "GTSO: Global Trace Synchronization and Ordering Mechanism for
Wireless Sensor Network Monitoring Platforms", Sensors, Vol. 18, No. 28, pp. 1-22, 2018.

[16] H. Karl and A. Willig, "Protocols and Architectures for Wireless Sensor Networks", Wiley-Blackwell, 2005.

[17] O. Demigha, N. Badache, M. Aissani and A. Mellouk, "Fault-Tolerant Prediction-based Scheme for Target Tracking Application", Proceedings of IEEE Global Telecommunications Conference, pp. 1-6, 2009.

[18] J. Tapeiro, H. Medeiros and R.H. Bishop, "Predicting Multiple Target Tracking Performance for Applications on Video Sequences", Machine Vision and Applications, Vol. 28, No. 5, pp. 539-550, 2017.

[19] N. Liang, G. Wu and W. Kang, "Real-Time Long-Term Tracking with Prediction-Detection-Correction", IEEE Transactions on Multimedia, pp. 1-14, 2018.

[20] D. Malan, T. F. Jones, M. Welsh and S. Moulton, “An Ad Hoc Sensor Network Infrastructure for Emergency Medical Care", Proceedings of International Workshop on Wearable and Implantable Body Sensor Networks, pp. 1-5, 2004.

[21] D. Wajgi and N. Thakur, "Load Balancing Algorithms in Wireless Sensor Network: A Survey", International Journal of Computer Networks and Wireless Communications, Vol. 2, No. 1, pp. 2250-3501, 2012.

[22] H. Tahmasebirad, "Target Tracking based on Dynamic Clustering with Energy Saving in Wireless Sensor Networks", Master Thesis, Department of Computer Science, Islamic Azad University, 2012.

[23] A. Ghazi and A. Ahiod, "Impact of Random Waypoint Mobility Model on Ant-based Routing Protocol for Wireless Sensor Networks", Proceedings of International Conference on Big Data and Advanced Wireless Technologies, pp. 1-7, 2016.

[24] M. Guo, E. Olule, G. Wang and S. Guo, "Designing Energy Efficient Target Tracking Protocol with Quality Monitoring in Wireless Sensor Networks", Journal Supercomputer, Vol. 51, No. 2, pp. 131-148, 2009.

[25] W. Heinzelman, A. Chandrakasan and H. Balakrishnan, "An Application-Specific Protocol Architecture for Wireless Microsensor Networks", IEEE Transactions on Wireless Communications, Vol. 1, No. 4, pp. 660-670, 2002.

[26] X. Ning and C.G. Cassandras, "Dynamic Sleep Time Control in Wireless Sensor Networks", ACM Transactions on Sensor Networks, Vol. 6, No. 3, pp. 1-37, 2010. 